\title{
Movement on pain
}

\author{
Tom Moberly UK editor
}

The BMJ

Doctors face the challenge of treating more people living more of their lives in ill health, and there is not a lot of good news around. For many people, current treatments for their long term conditions may not do much to relieve symptoms or improve their quality of life.

Fibromyalgia is one important example, featuring in The BMJ this week. At present, aerobic exercise is the most commonly prescribed non-drug treatment. Drug interventions, such as analgesics, seem to confer modest short term benefits but not much long term or clinically meaningful relief. In any case, many patients discontinue drugs either because they don't work or because of unpleasant side effects.

Complex pain disorders such as fibromyalgia may also contribute to opioid misuse. As with other pain disorders, many patients with fibromyalgia report using opioids to manage their pain, which carries the risk of dependency and misuse. This happens despite evidence that patients with fibromyalgia who receive opioids have poorer health outcomes than those given other treatments.

In this context, it is good news that tai chi, an ancient discipline rooted in traditional Chinese medicine, seems to be as effective as, or better than, aerobic exercise for managing fibromyalgia (doi:10.1136/bmj.k851). Importantly, the researchers found that patients were more likely to attend tai chi classes than the aerobic exercise sessions. "Patients may be more likely to enjoy, manage, and continue to practise tai chi, perhaps because it involves gentle, low impact movements with minimal side effects," says Chenchen Wang, one of the researchers, in a BMJ Opinion commentary explaining the study's context (http://blogs. bmj.com/bmj/2018/03/21/chenchen-wang-time-to-rethinkexercise-for-fibromyalgia-care).

Just as the best camera is said to be the one that you have with you, and the best medicine needs to be one that patients actually take, the best exercise programme may be the one that patients want to follow.

A word of caution about how these findings might be misappropriated is provided in a patient's perspective by Amy Price, who lives with chronic pain (http://blogs.bmj.com/bmj/ 2018/03/21/amy-price-the-unintended-consequences-of-tai-chifor-fibromyalgia). Price points out that tai chi doesn't work for everyone with fibromyalgia. "Helpful, cost effective interventions such as tai chi can be empowering as long as they are not used to deny other effective, but more costly, care," she says. 